\section{ONOMÁVAEIN}

Revista semestral de lingüística, filología y traducción
PONTIFICIA UNIVERSIDAD

\title{
La asociación de recursos de valoración: el caso de las organizaciones de derechos humanos en la prensa escrita argentina
}

Combining Appraisal resources: human rights

organizations in the Argentine press

\author{
Mariana Pascual \\ Universidad Nacional de San Luis \\ Argentina
}

\section{(c) (i) $\bigodot_{\mathrm{BY}}$}

Mariana Pascual: Facultad de Ciencias Humanas, Área Lenguas Extranjeras. Universidad Nacional de San Luis. Argentina Correo electrónico: pascualm@unsl.edu.ar 


\section{Resumen}

En las últimas décadas hemos presenciado un creciente interés por los estudios del lenguaje desde perspectivas críticas, fundados en la necesidad de develar posicionamientos ideológicos que atentan, a menudo, contra los intereses de grupos de limitado poder social. A fin de develar los posicionamientos, y en consecuencia la construcción y consolidación de ideologías a lo largo del tiempo, la teoría de la valoración (Martin, 2000; Martin y White, 2005), en el marco de la Lingüística Sistémico Funcional (LSF), brinda herramientas de particularvalor en el análisis de discursos de medios. Este marco se combina y enriquece con propuestas que comparten la base que responden a los postulados del enfoque sociohistórico discursivo propuesto por Reisigl y Wodak (2001) y por Wodak y Meyer (2003). La visión del lenguaje como práctica social (Fairclough y Wodak, 1997) es compartida por la LSF, en tanto esta se constituye como teoría lingüística multifuncional al servicio del análisis del discurso como entidad construida por los propios propósitos sociales a los que contribuye, y visualiza al lenguaje como una forma de semiótica social (Halliday, 1978; Halliday y Matthiessen, 2014). Este trabajo propone ilustrar el uso estratégico de recursos evaluativos y su asociación a partir del análisis de noticias publicadas en cinco influyentes diarios argentinos. Todos los artículos fueron publicados con motivo de un hecho de violencia de gran impacto social acaecido a me- diados de 1985, época marcada por el retorno de la democracia al país, en medio de una marcha militar liderada por el entonces presidente Alfonsín. En este hecho se vio involucrada la agrupación Madres de Plaza de Mayo, grupo formado por madres de las más de 30.000 víctimas de la dictadura militar que gobernó Argentina entre 1976 y 1983. El análisis arroja luz sobre el uso de una amplia variedad de recursos valorativos, tanto de Compromiso como de Actitud, así como también un uso vasto de elementos de Gradación, tanto infundidos lexicalmente como de formas aisladas. Resulta de particular interés la combinación de recursos valorativos realizada por los autores para la construcción de valoraciones de polaridad negativa acerca de las nociones de violencia y de disrupción de la normalidad. Sin embargo, las mayores diferencias observadas no fueron detectadas en los tipos de recursos léxico-gramaticales utilizados, sino en las diferentes intensidades de las evaluaciones planteadas. Este fenómeno podría dar cuenta de la imposibilidad de recurrir a otras opciones de construcción discursiva mediática viables que resulten políticamente correctas en un momento sociohistórico dado, como el que se analiza. Los resultados pueden contribuir a la comprensión del funcionamiento de los recursos lingüísticos por los que se construyen y consolidan posicionamientos ideológicos en la sociedad moderna mediatizada.

Palabras clave: teoría de la valoración; discurso de medios; agrupaciones de derechos humanos.

\section{Abstract}

The last decades have witnessed a growing interest in language studies from critical perspectives, aiming at revealing ideological positioning which often oppose the interest of groups with limited social power. With the aim of unveiling positioning, Appraisal Theory (Martin, 2000; Martin \& White, 2005) within Systemic Functional Linguistics (SFL) propo- ses analytical tools, which have proved to be particularly useful for the study of media discourse. This study illustrates the strategic use and combination of evaluative resources by analyzing the news stories published by five influential Argentinean newspapers regarding the organization Madres de Plaza de Mayo, which is a group formed by the mothers of over 30,000 
victims of the military dictatorship that ruled the country between 1976 and 1983 . The texts were published in relation to one specifically significant event that took place in 1985, in which a violent riot emerged in a military parade. The analysis sheds light on the combination of evaluative resources for the construction of negative values in relation to the judgment of violence and the disturbance of normality. However, the main difference lies not on the value constructed but on the intensity of the evaluation, which may be related to the lack of a politically correct option at a given sociohistorical point in time such as the one under analysis. The results may contribute to an understanding of the functioning of linguistic resources through which ideological alignment is constructed and consolidated in our modern mediatized society.

Keywords: Appraisal Theory; media discourse; Human Rights organizations.

\section{Introducción}

Durante las últimas décadas, los estudios del discurso desde perspectivas críticas han concentrado gran interés en el modo en que actores y procesos sociales son representados en los medios, fundados en la necesidad de develar posicionamientos ideológicos que atentan, a menudo, contra los intereses de grupos de limitado poder social.

El discurso de los medios guarda una estrecha relación con el desarrollo de la historia de las sociedades, ya que, debido a su papel cada vez más fundamental en la formación y consolidación ideológica, los medios masivos se han convertido en poderosos agentes sociales a la hora de determinar y fomentar el desarrollo de posicionamientos y alineamientos ideológicos. Tanto es así que algunos periodistas consideran que su producción en los medios sobre la historia reciente o historia actual actúa como "un primer borrador de la historia" (Garton Ash, 2000: 22), implicando que esa primera versión ha de ser eventualmente reformulada, reinterpretada y reconstruida con el paso del tiempo por una multiplicidad de actores sociales.

Dado este papel social de relevancia incuestionable, el análisis del discurso de medios realiza grandes aportes orientados a la comprensión de la manera en la que los recursos lingüísticos operan para construir posicionamientos ideológicos. A tal fin, la teoría de la valoración (Martin, 2000; Martin y White, 2005), como desarrollo de la Lingüística Sistémico Funcional (Halliday, 1978;
Halliday y Matthiessen, 2014), brinda herramientas de particular valor proponiendo el análisis sistemático de los recursos lingüísticos evaluativos.

Este marco puede ser combinado y enriquecido con propuestas teórico-analíticas que responden a los postulados del enfoque sociohistórico discursivo propuesto por Reisigl y Wodak (2001), Wodak (2001) y Wodak y Meyer (2003), quienes asignan aún más enfáticamente la centralidad de los aspectos sociohistóricos en los procesos de construcción y representación de los eventos sociales en diversos géneros discursivos. Comparten, al igual que la LSF, la visión del lenguaje como práctica social (Fairclough y Wodak, 1997) en tanto este marco se constituye como teoría lingüística multifuncional al servicio del análisis del discurso como entidad construida por los propios propósitos sociales a los que contribuye, y visualiza al lenguaje como una forma de semiótica social (Halliday, 1978).

Sobre el supuesto teórico de que el impacto evaluativo se construye en el discurso a partir de la combinación de estrategias, este trabajo propone ilustrar el uso estratégico de recursos evaluativos a partir del análisis de noticias publicadas en cinco diarios influyentes argentinos, de gran tirada en su área de cobertura: La Capital de la ciudad de Rosario, Los Andes de Mendoza, La Nación de Buenos Aires, El Tribuno de Salta y el diario Río Negro de la Ciudad de General Roca, en la región patagónica. Los artículos fueron publicados con motivo de un hecho de violencia de 
relativo impacto social acaecido a mediados de 1985, época marcada por el retorno de la democracia al país, en medio de una marcha militar liderada por el entonces presidente Raúl Alfonsín. En este evento se vio involucrada la agrupación Madres de Plaza de Mayo, grupo de defensa de derechos humanos conformado a partir de una de las situaciones más traumáticas de la historia reciente del país, la desaparición forzosa de más de 30.000 individuos, en su gran mayoría jóvenes, durante la dictadura militar que gobernó la Argentina entre 1976 y 1983.

\section{Fundamentación teórica}

\subsection{La teoría de la valoración y la prosodia evaluativa}

Una lectura exploratoria de los artículos que se analizan en este trabajo nos llevó a un supuesto teórico que surge de un primer acercamiento a los textos: ante fuertes presiones sociohistóricas de asumir un determinado posicionamiento, emerge en el discurso una evidente diferencia en intensidad del posicionamiento. En un momento de recuperación de democracia, de gran fervor social y pasión por la defensa de los derechos humanos, los medios de comunicación no podían más que acompañar a ese sentimiento popular, por causas de diversa índole, desde históricas hasta económicas. Sin embargo, las diferencias en los posicionamientos resultaba evidente, y esto nos condujo a indagar sobre la manera en la que se construyen las diferencias de intensidad de los posicionamientos, para establecer, en el caso de estos textos, a qué obedecían.

A tal fin, recurrimos a la teoría de la valoración como marco teórico-analítico, no solo porque ha demostrado resultar de gran utilidad en investigaciones anteriores (Oteíza, 2003, 2009a, 2009b, 2011; Oteíza y Pinto, 2008; Pascual, 2010, 2011, 2014, entre muchos otros), sino porque es el marco que proporciona la posibilidad de análisis entre diferentes tipos de significados, tanto interpersonales como textuales e ideacionales, y su propagación a lo largo del texto de manera prosódica (Martin y White, 2005). Estos autores sostienen que el análisis léxico-semántico de los significados evaluativos se funda en tres sistemas principales (ACTITUD, COMPROMISO y GRADACIÓN), que hacen referencia a los recursos semánticodiscursivos mediante los cuales se expresan apreciaciones y sentimientos desde una perspectiva inter- e intrapersonal. Esta teoría se ocupa de los recursos que posicionan de manera interpersonal las propuestas y proposiciones de los emisores (Martin y White, 2005). En otras palabras, según esta teoría, ciertos recursos lingüísticos construyen significados que hacen variar los planos del compromiso del hablante con sus emisiones, tanto individuales como acumulativas, que se presentan a medida que el texto se va desplegando.

En el contexto teórico general de la LSF, el proceso de producción del enunciado conjuga la elección de los significados y su estructuración, lo cual se lleva a cabo simultáneamente en tres planos de significado que Halliday y Matthiessen (2014) denominan metafunciones: la función ideacional (plano de contenido), la función textual (plano del texto mismo) y la función interpersonal (plano de la interacción). La teoría de la valoración profundiza el estudio de la función interpersonal, a través de la cual se manifiesta la interacción social y la expresión de nuestros puntos de vista sobre eventos y personas. La figura 1 ilustra el abanico de posibilidades organizadas en tres sistemas principales que ofrece la teoría de la valoración para analizar textos desde una perspectiva interpersonal.

Esta teoría plantea la organización de los significados en tres grandes dominios semánticos: ACTITUD, COMPROMISO y GRADACIÓN. A modo de muy breve síntesis, expondremos que la ActiTud constituye un aporte de singular relevancia a los estudios de significados, en tanto permite conocer e interpretar expresiones de valoración positiva o negativa respecto de personas, lugares, objetos, hechos y circunstancias. De los tres 


\section{FIGURA 1}

La teoría de la valoración (Martin y White, 2005: 38)

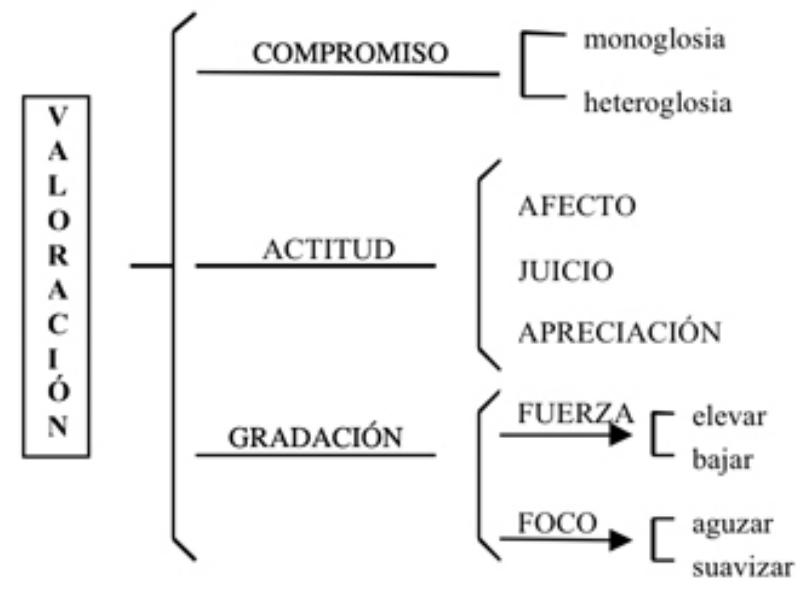

subsistemas de Actitud ${ }^{1}$ (emocional [Afecto], ético [Juicio] y estético [Apreciación]), el presente trabajo indaga acerca de cómo los tres tipos de significado se combinan en un interjuego de intensidades. Toma como punto de partida para el análisis interpersonal el Juicio, característico de la construcción de nuestra estimación del carácter de la gente y la evaluación de la ética de su conducta, el cual se divide en Sanción Social y Estima Social. El siguiente ejemplo² ilustra una clara instancia de Juicio:

La plaza es de las Madres y no de los cobardes ${ }^{3} .$. (La Capital)

Aquí los autores presentan un Juicio, en cuanto evalúan el carácter y el accionar de los individuos como cobardes, haciendo uso de una realización asociada a la Tenacidad, como subcategoría de la Estima Social, de carácter negativo.

Otro de los sistemas es el de Afecto, asociado a las emociones, que incluye cómo nos sentimos y cómo reaccionamos frente a los fenómenos que percibimos y los deseos de cosas que que- remos, como lo ilustra el siguiente ejemplo, en el cual el Afecto se presenta en combinación con la exteriorización física que produce, gritos:

Al iniciarse el desfile, los integrantes de la columna comenzaron a gritar "asesinos, asesinos") (Los Andes)

El tercer sistema de la Actitud es la Apreciación, que guarda relación con la evaluación de objetos y productos vinculada a principios estéticos y otros sistemas de valor social (Martin y White, 2005). Este tipo de evaluación puede realizarse en términos de su Composición, Reacción o Valor. La valoración en términos de Composición se refiere a la percepción, a nuestra visión del orden (por ejemplo: equilibrado, armonioso); la Reacción se relaciona con el afecto en cuanto expresión desiderativa o emotiva (por ejemplo: atrapante, aburrido), mientras que la Apreciación expresada por un recurso de Valor se asocia a la cognición (por ejemplo: creativo, convencional).

Por otro lado, el sistema de сомрromiso, que también se analiza en el presente trabajo, comprende los recursos lingüísticos para posicionar la voz del autor en relación con las propuestas y proposiciones de un texto. Este sistema comprende dos grandes subsistemas, Monoglosia y Heteroglosia. En el caso de la Monoglosia los autores no incorporan otras voces en el discurso. La Heteroglosia, por otra parte, se relaciona con el dialogismo, la polifonía y la intertextualidad, y puede subcategorizarse en extravocalización o expansión dialógica, e intravocalización o contracción dialógica ${ }^{4}$. Estos significados se relacionan con los principios de contracción o expansión de los espacios dialógicos, y resultan de particular utilidad al analizar cómo los medios incluyen o excluyen ciertas voces, ciertos participantes, lo cual constituye un recurso fundamen-

1 Siguiendo la tradición de los estudios de la LSF, los nombres de los sistemas de la teoría se indican en versalita (small caps).

2 Los ejemplos presentados han sido extraídos de los textos analizados.

3 Se indican con subrayado las formas de inscripción de la valoración.

4 Si bien los términos “intravocalización” y "extravocalización” no son propuestos en la teoría original por Martin y White (2005), han sido adoptados en numerosos estudios de valoración de América Latina como en los de Kaplan (2004), Oteíza (2009a) y los de Achugar y otros (2011), entre muchos otros, para hacer referencia al cierre y apertura del espacio dialógico, respectivamente. 
tal en la comunicación periodística. El siguiente ejemplo ilustra la heteroglosia del discurso en la incorporación en forma de Reconocimiento en el hecho que se reporta, en este caso, la voz de los civiles:

... el grupo de civiles atronaba con la consigna "Alfonsín, Alfonsín”. (Los Andes)

En lo que respecta al tercer sistema, la GRADACIÓN, comprende los recursos que el lenguaje ofrece para intensificar o mitigar los significados de ACTITUD o compromiso, así como también para la gradación de significados ideacionales. Incluye dos subsistemas principales: la Fuerza y el Foco. Por medio de los recursos de Fuerza, tanto aislados como fusionados lexicalmente, se gradúan las evaluaciones en términos de Intensificación o de Cuantificación. El otro subsistema es el de Foco, por medio de cuyos recursos se acentúan o atenúan los límites de las entidades, opiniones, sentimientos, posicionamientos, como lo ilustra el siguiente ejemplo:

... un individuo... le pegó duramente a un adolescente que lo increpaba. (La Capital)

Por otra parte, esta teoría resulta de particular utilidad al considerar otros recursos que no operan de modo aislado en el discurso, en lo que Martin y White (2005) denominan el fenómeno de la prosodia, por medio del cual las evaluaciones del discurso van gradualmente expandiéndose, potenciándose en procesos de resonación que trascienden los límites lexicales, como lo demuestra el siguiente ejemplo extraído del diario La Capital:

Al terminar el desfile se produjo otro violento incidente entre los acompañantes de las Madres y militantes de la juventud radical. Uno de los integrantes del grupo de los derechos humanos fue golpeado en la esquina de la diagonal Julio A Roca y Perú, a escasos metros de la plaza. El enfrentamiento se desencadenó cuando después de un intercambio de insultos entre ambos grupos un individuo con campera amarilla que llevaba en su pechera un distintivo rojiblanco le pegó duramente a un adolecente que lo increpaba.
Como reacción inmediata, integrantes de los dos bandos enfrentados se abalanzaron e intercambiaron golpes de puños y puntapiés, cayendo al suelo uno de los hombres que acompañaba a las Madres. En la confusión, un periodista del matutino "El Cronista Comercial", Andrés Sikirko, fue agredido de un puñetazo en el mentón por un joven que se identificó como militante de la juventud peronista, pero que llevaba en su pechera un distintivo radical.

La intensidad de la evaluación se hace evidente por la reiteración a lo largo del párrafo de un gran número de expresiones relacionadas con la violencia en sus diferentes formas. Asimismo, el grado de detalle que surge de la presentación ideacional de los hechos suma dramatismo, y construye prosodia en relación con el evento presentado.

Otro concepto valioso que aporta al análisis que se presenta en este trabajo es el de densidad evaluativa (DE). Esta noción ha sido definida y aplicada en numerosos estudios previos, fundamentalmente los de Shiro (1998, 2003), Kaplan (2004) y muchos otros autores, para estimar la frecuencia de uso de términos evaluativos en el discurso. Es una noción particularmente útil al comparar intensidades de posicionamientos entre diferentes textos. Si bien creemos que no es un indicador valioso si se lo utiliza de manera aislada, puede contribuir al análisis de intensidades evaluativas como el que presentamos, constituyéndose en un factor más de variación en intensidades evaluativas.

En línea con estas conceptualizaciones y siguiendo a White (1998), coincidimos en que la evaluación muestra una funcionalidad polisémica dependiente del contexto, por lo cual la incorporación de un fuerte anclaje sociohistórico (Wodak, 2001; Reisigl y Wodak, 2001) se constituye en un requisito imperioso para el tipo de análisis que aquí exponemos. El enfoque sociohistórico discursivo fue inicialmente construido para el análisis de las imágenes estereotipadas antisemitas; sin embargo, su aplicación se ha diseminado ampliamente y su aplicación ha llega- 
do a alcanzar una enorme variedad de géneros, ideologías y contextos.

\subsection{El discurso de la historia reciente}

La historia reciente forma una parte fundamental de la identidad social y su estudio ha atraído a un gran número de académicos desde variadas perspectivas. En el campo de los estudios del lenguaje, la investigación que aquí presentamos tiene como antecedentes una larga lista de trabajos relacionados de analistas discursivos de Chile, Uruguay y Argentina (Achugar, 2008, 2009; Achugar y otros, 2011; Achugar y Schleppegrell, 2005; Oteíza, 2003, 2009a, 2009b, 2011; Oteíza y Pinto, 2008, 2011; Giudice, 2010; Giudice y Moyano, 2011; Zullo, 2012, 2014, entre otros). A fines del siglo pasado, tanto Argentina como Chile y Uruguay fueron víctimas de dictaduras que, en mayor o menor grado, basaron su accionar en prácticas de exterminio de opositores, con desaparición forzada de personas, censura, coacción, tortura física y psicológica, y otras numerosas actividades relacionadas al terrorismo de Estado.

Sin embargo, la mayoría de los trabajos anteriormente citados, fundamentalmente los desarrollados por Oteíza, Giudice y Zullo, se han basado primordialmente en el análisis de los recursos representacionales construidos en materiales de enseñanza de diferentes niveles de educación; es decir, indagan acerca de la construcción del pasado reciente a partir de materiales didácticos, principalmente libros de texto y manuales escolares. Por otra parte, el trabajo de Achugar y colaboradores se focaliza en la legitimación de la violencia a partir del análisis del discurso militar, de los manuales de historia y, más recientemente, de artefactos culturales multisemióticos, tales como canciones, programas de televisión y documentales.

A la fecha, si bien se han realizado análisis de la historia reciente de la Argentina por parte de investigadores de otras disciplinas, como la historia o la sociología, los analistas discursivos no se han concentrado en el estudio de esta temática en géneros mediáticos, como lo propone la presente investigación.

\subsection{El discurso de los medios: la noticia}

Siguiendo la propuesta de Martin y Rose (2008), consideramos a la noticia como un representante de la familia de géneros de tipo narrativo (story genres), en la cual se intenta cumplir con el propósito social de distribución, diseminación y consolidación de valores culturales. A diferencia de otros géneros textuales como los de la ciencia, la religión y muchas otras esferas de la práctica social, los géneros mediáticos, y en particular la noticia, se caracterizan por su naturaleza multiautoral. En su construcción participan numerosos autores, quienes, a su vez, se constituyen en audiencias intermedias. Resulta innegable que la responsabilidad final del contenido proposicional de la noticia es o al menos debería ser asumida por los propietarios de los medios, quienes ejercen un poder mayor sobre el contenido publicado. El siguiente gráfico expone esquemáticamente los diferentes autores y el papel que juegan en la construcción del discurso de la noticia:

\section{GRÁFICO 1}

La construcción de la noticia (de Feez y otros, 2008: 57)
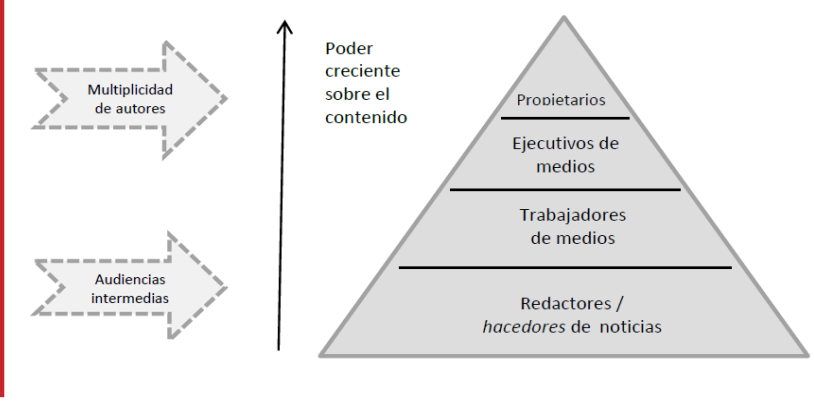

Enumerar los antecedentes de análisis de los medios trasciende los límites y objetivos del presente trabajo, pero resulta digno de destacar que esta visión de la noticia como texto que 
surge de la comunión de autores, en un acto de construcción de multiplicidad de significados en el marco de un contexto claramente situado sociohistóricamente, nos posibilita arrojar luz sobre cómo operan los recursos lingüísticos para la construcción y consolidación discursivas de ideologías

\section{El contexto sociohistórico}

\subsection{La Asociación Madres de Plaza de Mayo (AMPM)}

Durante la última dictadura militar, y en línea con lo que ocurría en otras latitudes de Latinoamérica, se crearon y consolidaron organizaciones de ciudadanos dedicadas a luchar por la plena vigencia de los derechos humanos frente al terrorismo de Estado. Entre ellas, la Asociación Madres de Plaza de Mayo se destacó como ícono de lucha, sufrimiento y perseverancia. Se constituyó espontáneamente en abril de 1977, cuando, unidas por la soledad, la confusión y el dolor por la pérdida de sus hijos, deciden convocarse para enfrentar juntas la desolación. Comenzaron a hacer búsquedas organizadas en comisarías, hospitales, morgues y en toda institución que pudiese ayudarlas a unirse a sus hijos. Luego comenzaron a movilizarse para expresar su descontento de modo público con las famosas marchas que desde entonces y hasta la actualidad se desarrollan todos los jueves en la histórica Plaza de Mayo de Buenos Aires. La asociación ha recibido el reconocimiento internacional de numerosos organismos de derechos humanos (Vázquez, 2009).

Disidencias internas sobre los niveles de participación política, las metodologías, los propósitos perseguidos y fundamentalmente el personalismo de la líder histórica, Hebe Pastor de Bonafini, hicieron que la asociación se fragmentara en 1986. Fue por entonces que se constituyeron dos líneas fundamentales de la organización: Madres de Plaza de Mayo, presidida por Bonafini, y Línea Fundadora, liderada por Marta Ocampo de Vásquez. Los textos analiza- dos aquí corresponden a una etapa en la cual el movimiento no se había fragmentado aún, sino que estaba inmerso en una lucha cotidiana por consolidar su identidad como asociación, frente a las embestidas de los medios más conservadores que sistemática e inevitablemente las asociaban a la violencia extrema y al terrorismo.

\subsection{Argentina y la dictadura militar}

Entre el 24 de marzo de 1976 y el 10 de diciembre de 1983 la Argentina estuvo gobernada por juntas militares. Tomaron el gobierno luego de que en julio de 1973 muriera Juan D. Perón y la presidencia quedase en manos de su esposa, María Isabel Martínez, quien demostró una incapacidad extrema para conducir los destinos del frágil país. Este hecho marcó el inicio de años de inestabilidad política y económica que llevaron al golpe militar. La primera junta militar estaba compuesta por Videla, Massera y Agosti, representando a la Fuerza Aérea, la Marina y el Ejército, respectivamente. La junta militar protagonizó lo que se conoce en la historia del país como “la Guerra Sucia”, una sucesión despiadada de hechos de crueldad inédita cuyo objetivo era la aniquilación por vía de la represión de todas las líneas opositoras. Como consecuencia de la privación ilegítima de la libertad y de la desaparición forzosa, 30.000 individuos murieron. Se estima que aproximadamente $80 \%$ de ellos no estaban de modo alguno relacionados con la subversión y que en el primer año de dictadura ya había más de 15.000 desaparecidos, 10.000 presos, 4.000 muertos y decenas de miles de exiliados.

Ante semejante panorama, surgen numerosas instituciones tendientes a proteger el orden social, la restauración y permanencia de las instituciones democráticas y los derechos humanos. Nacen entonces, entre otros, la Asociación Madres de Plaza de Mayo. No resultó fácil armonizar el retorno a la democracia en 1983 en medio de ese contexto social, y el papel que jugaron los medios fue crucial. 


\section{Criterios de selección de textos y análisis}

Para este trabajo se seleccionaron 5 textos, extraídos de un corpus mayor, de 5 diarios argentinos, los cuales ejercen una gran influencia en su zona de cobertura y pertenecían a diferentes grupos mediáticos o empresas familiares al momento de publicación. Todos los textos pertenecen al género noticias de interés humano (Feez y otros, 2008). Fueron seleccionados por constituir ejemplares que ilustran cómo el mismo evento puede ser construido de maneras distintas, haciendo uso de recursos evaluativos que materializan cuerpos de creencias ideológicas diferentes. Todos los textos analizados fueron publicados en igual fecha, el 10 de julio de 1985, día inmediatamente posterior al del evento construido: los actos de violencia ocurridos durante el desfile en conmemoración de la Independencia, presidido por Raúl Alfonsín. La tabla 1 muestra las características principales de los textos analizados.

Como puede observarse, las noticias seleccionadas están compuestas por una cantidad de palabras en un rango que varía entre un mínimo de 553 (T1-ET)5 y un máximo de 1172 (T4-LN), presentan variaciones en términos de organización de la información bajo subtítulos y también difieren en la agencia que convirtió el evento en un hecho noticioso, en tanto 2 artículos se originaron en la agencia DyN (T2-LA y T5-RN), otros 2 en NA (TI-ET y T3-LC) y uno es de redacción propia (T4-LN). Cabe mencionar que ninguno de los artículos mencionados lleva la firma de un autor, sino que, como es la práctica usual en este género - noticias de interés humano-y a diferen-

\section{TABLA 1}

Características de los textos seleccionados para análisis

\begin{tabular}{|c|c|c|c|c|c|c|}
\hline & Diario & Ciudad & Titular & Subtítulos & $\begin{array}{l}\text { Agencia } \\
\text { noticiosa }\end{array}$ & $\begin{array}{l}\text { Número de } \\
\text { palabras }\end{array}$ \\
\hline 1 & $\begin{array}{l}\text { El } \\
\text { Tribuno }\end{array}$ & Salta & $\begin{array}{l}\text { Incidentes en } \\
\text { Plaza de Mayo }\end{array}$ & Ninguno & $\begin{array}{l}\text { NA } \\
\text { Noticias } \\
\text { Argentinas }\end{array}$ & 553 \\
\hline 2 & $\begin{array}{l}\text { Los } \\
\text { Andes }\end{array}$ & Mendoza & $\begin{array}{l}\text { Hubo serio incidente } \\
\text { con Madres de } \\
\text { Plaza de Mayo }\end{array}$ & Ninguno & $\begin{array}{l}\text { DyN } \\
\text { Diarios y Noticias }\end{array}$ & 567 \\
\hline 3 & La Capital & Rosario & $\begin{array}{l}\text { Dos lesionados como } \\
\text { saldo de incidentes } \\
\text { ocurridos antes y } \\
\text { después del desfile }\end{array}$ & $\begin{array}{l}\text { (2) - Violento Incidente } \\
\text { - Intercambio de Insultos }\end{array}$ & $\begin{array}{l}\text { NA } \\
\text { Noticias } \\
\text { Argentinas }\end{array}$ & 567 \\
\hline 4 & La Nación & $\begin{array}{l}\text { Buenos } \\
\text { Aires }\end{array}$ & $\begin{array}{l}\text { Hubo incidentes } \\
\text { con las Madres de } \\
\text { Plaza de Mayo }\end{array}$ & $\begin{array}{l}\text { (3) - Denuncia } \\
\text { - "Jornada Bochornosa" } \\
\text { - La seguridad no funciona }\end{array}$ & $\begin{array}{l}\text { Redacción } \\
\text { propia }\end{array}$ & 1172 \\
\hline 5 & Río Negro & Río Negro & $\begin{array}{l}\text { Incidentes entre } \\
\text { las Madres y un } \\
\text { grupo de civiles }\end{array}$ & $\begin{array}{l}\text { (1) - Las consignas } \\
\text { de las Madres }\end{array}$ & $\begin{array}{l}\text { DyN } \\
\text { Diarios y Noticias }\end{array}$ & 705 \\
\hline
\end{tabular}

5 Se determina la siguiente codificación de los textos (T): enumeración de 1 a 5, según el orden alfabético de las iniciales del medio de publicación y separados por un guion de las iniciales del medio. Los códigos resultantes son: T1-ET = texto 1, diario El Tribuno; T2-LA = texto 2, diario Los Andes; T3-LC = texto 3, diario La Capital; T4-LN = texto 4, diario La Nación; T5-RN = texto 5, diario Río Negro. 
cia, por ejemplo, de las noticias de opinión o de las editoriales, la autoría se atribuye al medio y a la agencia noticiosa exclusivamente.

Los 5 textos fueron analizados en su totalidad, utilizando las herramientas analíticas de los sistemas de Apreciación. Asimismo, se calculó la DE de cada uno de los textos y la prosodia que la combinación de recursos generaba ${ }^{6}$.

\section{El uso de las estrategias de valoración}

Presentamos los resultados del análisis en relación con las categorías básicas introducidas: ACTITUD, COMPROMISO y GRADACIÓN. También incluimos resultados relativos a la $D E$, como elemento indicador de intensidades evaluativas, y breves consideraciones acerca de la combinación de recursos y generación de prosodia evaluativa.

\subsection{La ACTITUD, el COMPROMISO y la GRADACIÓN}

El análisis de recursos de Actitud demostró que los autores de todos los textos hicieron un uso de estrategias similares, con clara predominancia en la totalidad de los casos de valores de Juicio, en abundantes críticas de tipo sanción social, en la forma de cuestionamientos a la integridad, que se expresan desde el eje negativo de la polaridad. Estas estrategias son esperables, en tanto condenan la violencia, a la vez que, en contraste, también se utilizan valores de Juicio relacionados con la normalidad, veracidad y la paz social, en los extremos positivos. Al tratarse de textos de un género mediático cuya intención es la de "la utilización de un evento con el propósito de ejemplificar un valor social o moral en particular" (Feez y otros, 2008: 136), la predominancia de los valores de Juicio establece una pertenencia y lo define como ejemplar del género discursivo al que pertenece: noticia de interés humano

Algunos ejemplos de esta sanción social incluyen:

1. La policía les impidió ingresar al paseo para exteriorizar sus reclamos. (T2-LA)

2. Los manifestantes entonaron permanentemente consignas tales como "La plaza es de las madres y no de los cobardes". (T2-LA)

3. "Por los 30.000 desaparecidos, exigimos juicio y castigo a los culpables". (T3-LC)

4. Fue una ardua tarea para el personal policial ubicado en el lugar, mantener el limite mientras los manifestantes gritaban gruesos insultos, "hijos de p..., asesinos". (T4-LN)

5. Algunos manifestantes comenzaron a correr a los jóvenes, mientras las Madres intentaban imponer calma... (T5-RN)

Como se puede observar, algunas evaluaciones construyen juicios positivos, como en el ejemplo 5, en el cual se construye a las Madres como generadoras de "calma", pero predominan los juicios negativos, y tienen como entes evaluados a la policía o a algunos militantes, pero mayormente el objetivo de la evaluación negativa son los militares responsables de los abusos ocurridos durante la dictadura. Eso resulta incuestionable, es ese el propósito social que en última instancia los textos tratan de consolidar: un rechazo a los excesos, a la violencia, y una consolidación de valores democráticos. Ahora bien, las evaluaciones difieren en frecuencia, como se verá en el análisis de DE, y en carga valorativa.

La tabla 2 resume los resultados en términos de la cantidad de instancias identificadas por medio para cada una de las categorías de ACTITUD, COMPROMISO y GRADACIÓN, así como también las cifras

6 Utilizaremos aquí una versión modificada de la propuesta originalmente por Shiro (1998). En esta nueva versión la DE se estima de la siguiente manera: tas como evocadas; el número de palabras corresponde al número de palabras por texto, y la cifra 1000 al valor de normalización adoptado para validar la comparación entre textos. 
de DE registradas para cada texto, a ser consideradas en la próxima sección.

Como lo demuestra la tabla 2, en contraste con las de Juicio, las instancias de Apreciación y Afecto fueron mínimas. Sin embargo, debemos destacar la presencia de valores en el eje del Afecto, que no constituye una característica típica de este género. Los recursos de Afecto identificados se referían principalmente a reacciones emocionales vinculadas con la expresión física de infelicidad e insatisfacción, como lo demuestran los siguientes casos, 6 y 7, extraídos de La Nación y del diario Río Negro, respectivamente:

6. Schiller respondió: "eso es un cuento que hoy agitan los partidarios de las fuerzas armadas que reprimieron al pueblo", "yo no puedo permitir - expresó luego-que los asesinos se paseen libremente con mis hijos". (T4-LN)

7. Al iniciarse el desfile, los integrantes de la columna comenzaron a gritar: "asesinos, asesinos". (T5$\mathrm{RN})$

En el ejemplo 6, se observa una codificación de Afecto de tipo irrealis, que construye deseo, en este caso, el deseo de justicia. A su vez, en el ejemplo 7 observamos que por medio de los gritos de "asesinos, asesinos" se evoca una instancia de Afecto que codifica la infelicidad de las Madres y sus simpatizantes ante la presencia de los militares que desfilaban, a los cuales consideraban responsables por las desapariciones de sus hijos. La inclusión de estos valores coincide con los textos de mayor compromiso evaluativo.

\section{TABLA 2}

Frecuencia de ACTITUD, COMPROMISO y GRADACIÓN, y densidad evaluativa registrada (valor normalizado cada 1000 palabras).

\begin{tabular}{|c|c|c|c|c|}
\hline \multirow[b]{2}{*}{ Diario } & \multicolumn{3}{|c|}{ Recurso valorativo } & \multirow[b]{2}{*}{ DE } \\
\hline & Nro. de instancias de ACTITUD & $\begin{array}{l}\text { Nro. de instancias de } \\
\text { compromiso heteroglósico }\end{array}$ & $\begin{array}{l}\text { Nro. de instancias } \\
\text { de GRADACIÓN }\end{array}$ & \\
\hline T1-ET & $\begin{array}{c}31 \\
\text { (25 de Juicio y } 6 \text { de } \\
\text { Apreciación) }\end{array}$ & $\begin{array}{c}8 \\
\text { (4 atribuciones a MPDM) }\end{array}$ & 7 & 17,7 \\
\hline T2-LA & $\begin{array}{c}37 \\
\text { (31 de Juicio, } 5 \text { de } \\
\text { Apreciación, } 1 \text { de Afecto) }\end{array}$ & $\begin{array}{c}8 \\
\text { (5 atribuciones a MPDM) }\end{array}$ & 6 & 18,12 \\
\hline T3-LC & $\begin{array}{c}40 \\
\text { (36 de Juicio, 2 de } \\
\text { Apreciación, } 2 \text { de Afecto) }\end{array}$ & (7 atribuciones a MPDM) & 15 & 34,61 \\
\hline T4-LN & $\begin{array}{c}67 \\
\text { (52 de Juicio, } 9 \text { de } \\
\text { Apreciación y } 6 \text { de Afecto) }\end{array}$ & $\begin{array}{c}32 \\
\text { (23 atribuciones a MPDM } \\
\text { y sus simpatizantes) }\end{array}$ & 17 & 62,48 \\
\hline T5-RN & $\begin{array}{c}32 \\
\text { (26 de Juicio, 1 de } \\
\text { Apreciación, } 5 \text { de Afecto) }\end{array}$ & $\begin{array}{l}8 \text { heteroglosia ( } 5 \\
\text { atribuciones a MPDM } \\
\text { y sus simpatizantes) }\end{array}$ & 10 & 23,97 \\
\hline
\end{tabular}


Con relación al compromiso, y en consonancia con el género bajo análisis, la expansión dialógica se presentó principalmente en la forma de atribuciones y de reconocimiento de otras voces que son claramente identificadas. Este recurso opera para reforzar la veracidad de un texto y presenta la ventaja de una aparente protección de la voz autoral, al transferir la responsabilidad proposicional de modo directo o en forma de cita reportada a otras voces que se incorporan. Las formulaciones de cita directa presentan la proposición como verdadera o válida (Feez y otros, 2008). Si bien el análisis demostró que la mayoría de las aseveraciones fueron monoglósicas y se produjeron escasas codificaciones de la evaluación en esas instancias, los autores construyeron las valoraciones desde las voces introducidas de otros actores sociales. En el caso de los diarios La Capital, La Nación y Río Negro, la fuente de las atribuciones correspondió a miembros de la AMPM o a simpatizantes de este movimiento. En contraste, El Tribuno y Los Andes no solamente hicieron un uso más escaso de atribuciones, sino que, al hacerlo, abrieron el espacio para una variedad de actores sociales relacionados con el incidente, pero no con el objetivo de los grupos de apoyo a los derechos humanos, como puede observarse en los siguientes ejemplos:

8. Un grueso cordón policial impidió que las madres pudieran lograr su objetivo y uno de los jefes del operativo explicó a un cronista de Noticias Argentinas que la prohibición regía respecto a los carteles, los cuales no podían ser exhibidos en la plaza. (T1-ET)

9. Los civiles, algunos de los cuales les gritaron a los manifestantes "trotskos" y "váyanse", transponían sin dificultad el cordón policial que impedía el paso de las Madres. (T2-LA)

Con respecto a la GRADACIÓN, pudo observarse un uso frecuente de intensificaciones de las valoraciones, que son predominantemente realizadas por cuantificadores. El efecto intensificador se obtuvo de multiplicidad de modos, entre los que se destacaron cuantificadores, aproximantes, repeticiones lexicales, evaluaciones lexicalizadas y nivel de detalle muy específico en los significados ideacionales.

El ejemplo 10, que presentamos a continuación, ilustra el uso de detalles ideacionales como reforzadores de intensidad de la evaluación:

10. La formación policial fue reforzada por un pelotón de la guardia de infantería, con cascos y pistolas lanza gases, que se ubicó detrás del cordón y al lado de un carro de asalto. (T2-LA)

En este caso Los Andes construyó una evaluación negativa de la policía, como abusiva, agente de un accionar desproporcionado con respecto a las dimensiones del incidente, y el detalle ideacional refuerza esa idea.

La suma de estos recursos no solo le da la textura de los textos, sino que le brinda a la evaluación un carácter prosódico notable. A modo de ejemplo, los siguientes recursos utilizados en el artículo de Los Andes (T2-LA) actúan construyendo, reforzando o intensificando la posición de los autores ante los eventos?:

manifestantes que acompañaban a las Madres de Plaza de Mayo y un grupo de civiles los manifestantes -entre ellos medio centenar de Madres con sus clásicos pañuelos blancos

varios manifestantes, dos fotógrafos del periódico de Madres de Plaza de Mayo, un reportero gráfico de "El Cronista Comercial" y un redactor de ese medio.

un hombre ... otras dos personas

La mayoría de los que participaron en los enfrentamientos

Los civiles, algunos de los cuales les gritaron a los manifestantes

Los manifestantes, alrededor de doscientos

El paso de la pequeña columna

Los manifestantes

La formación policial fue reforzada por un pelotón de la guardia de infantería, con cascos y pis-

7 Se identifican con letra negrita las instancias de repetición y con sombra las cuantificaciones. 
tolas lanza gases, que se ubicó detrás del cordón y al lado de un carro de asalto.

\section{Los manifestantes}

los integrantes de la columna ... mientras que el grupo de civiles

Los intentos de uno y de otro lado

los manifestantes- muchos de los cuales llevaban sobre el rostro mascaras

Cabe aquí señalar que los textos de $\mathrm{La} \mathrm{Na}$ ción y La Capital mostraron mayor uso de elementos que intensifican las evaluaciones y también mayor variedad e intensidad en cuanto a los recursos utilizados, tanto de Juicio como de GRADACIÓN de los mismos.

Vemos así que este análisis es revelador en cuanto a los posicionamientos de cada medio en términos de los esfuerzos que invierten en codificar evaluaciones con respecto a la temática. Consideraremos ahora otros de los factores que esperamos arrojen luz sobre la comprensión de este fenómeno

\subsection{La densidad evaluativa}

La DE es uno de los elementos que pueden contribuir a la comprensión de las intensidades evaluativas. En el caso de los textos analizados, para estimarla consideramos la totalidad de instancias, tanto explícitas como invocadas, con los resultados mostrados en la tabla 2 . Como puede observarse, existen grandes variaciones entre las DE identificadas, que van desde un mínimo de 17,7 hasta un máximo de 62,46 instancias cada 1000 palabras para los diarios El Tribuno y La Nación, respectivamente. Estas cifras, si bien son indicadores meramente cuantitativos, dan cuenta de los esfuerzos de los medios por codificar evaluaciones - de diversa intensidad-, aunque a nuestro parecer su principal aporte es la identificación de aquellos medios que no construyen numerosas valoraciones sobre la temática, tales como el caso de El Tribuno y Los Andes, lo cual podría responder a una intencionalidad de distanciarse de la temática de los derechos humanos. Asimismo, resulta razonable suponer que, a mayor cantidad de instancias evaluativas, los medios codifiquen un compromiso mayor de consolidación de los valores sociales relacionados a la temática. Por otra parte, las altas cifras, por ejemplo, de La Nación, aunque pueden no ser un reflejo directo de su intensidad, dan cuenta de una preocupación de los autores de los textos por evaluar la realidad que construyen.

\subsection{La prosodia evaluativa}

Todos los elementos parecen indicar que la intensidad en la evaluación del posicionamiento se logra a partir de la combinación de una multiplicidad de recursos que el lenguaje ofrece en los procesos de construcción textual. Posiblemente la incorporación de uno o algunos pocos de esos recursos no resulte en tonos evaluativos tan evidentes. Sin embargo, existe una clara relación entre el uso frecuente y variado de recursos, lo cual redunda en altas densidades evaluativas, que construyen prosodia evaluativa en el caso de La Nación, La Capital y Río Negro, y que pueden asociarse a un interés de estos medios por construir un claro posicionamiento a favor de la temática planteada: el desprecio por la violencia ejercida sobre un grupo de lucha en favor de los derechos humanos. En claro contraste, los medios de bajas DE como El Tribuno y Los Andes, justamente por el escaso uso de recursos, no construyeron valoraciones que pudiesen generar prosodia $y$, en consecuencia, tampoco un posicionamiento contundente a favor de los valores sociales en juego, conducta que se asocia al conservadurismo y a los extremos de las derechas ideológicas (Wodak y otros, 2013).

\section{Conclusiones}

El análisis de estos textos nos ha permitido identificar algunas probables formas de construcción de intensidades evaluativas en el género noticias. La evidencia sugiere que el elevado tono surge a partir de la combinación de diferentes recursos, tanto ideacionales como interpersonales, que operan para generar prosodia y consolidar la posición ideológica de los autores con variables grados de intensidad, más allá de 
las presiones sociohistóricas que limitan las declaraciones abiertas en favor de una u otra línea ideológica.

Consistentemente, los medios más tradicionales, que se vinculan a valores conservadores de derecha (Wodak y otros, 2013), y que se resistían a las representaciones evaluativas de apoyo a la causa de los movimientos de derechos humanos, como el MPDM, tendieron a restringir la frecuencia de uso de recursos valorativos, y en consecuencia mostraron baja DE, como en el caso de El Tribuno y Los Andes. Asimismo, otro factor que demostró ser indicativo de la falta de apoyo sustancial a las causas de las MPDM fue la escasez de instancias de asignación de voz a estas asociaciones, que también se identificó en estos medios. A estos factores debemos agregar un tercer fenómeno identificado, el uso reducido de elementos de intensificación de la fuerza de las evaluaciones. La conjugación de estos tres factores -escaso uso de recursos valorativos (baja DE), escasa inclusión de las voces de estos actores sociales y un uso limitado de recursos intensificadores - parece ser determinante a la hora de construir un distanciamiento ideológico que denota la falta de apoyo a los valores sostenidos por las MPDM.

Por el contrario, los medios que construyeron posicionamientos contundentes en favor de los valores sociales relacionados con las Madres, los diarios La Nación, La Capital y Río Negro, lo hicieron por medio de un uso frecuente de recursos de valoración (alta DE), apertura del espacio dialógico del discurso a las voces de las Madres y un elevado número de instancias de intensificación de las valoraciones. Resulta de interés subrayar que no se observaron diferencias en cuanto a las agencias de producción de las noticias, lo cual sugiere que la impronta ideológica se otorga en el diario que lo publica y no en la agencia que le da un origen primero, confirmando así la idea de que, a medida que el texto original atraviesa las diferentes instancias de autoría, va adquiriendo un perfil de posicionamiento que es enteramen- te adjudicable al medio.

Algunas inquietudes que surgen de este trabajo y que esperamos poder develar en el futuro incluyen determinar si estos posicionamientos se sostienen a lo largo del tiempo. Desde el plano de la construcción del discurso, futuros estudios podrían determinar si algunos recursos son inherentemente más intensos que otros y si la intensidad de los tonos evaluativos depende de las configuraciones específicas de cada género. Otras posibles líneas de investigación incluyen comparaciones entre géneros, tales como los relativos a la historia y los de los medios, que, si bien presentan grandes diferencias, también han demostrado múltiples coincidencias.

La presente investigación, aunque realizada sobre la base de un número limitado de textos, dio cuenta de la combinación de recursos valorativos, la cual consideramos fundamental en la construcción del tono evaluativo del discurso. La comprensión de la evaluación en el lenguaje de los medios es un factor de suma importancia en la construcción de valores sociales en la vida moderna, cada vez más mediatizada.

\section{Bibliografía citada}

Achugar, Mariana, 2008: What we remember: The construction of memory in military discourse, en Discourse Approaches to Politics, Society and Culture Series (eds.: Ruth Wodak y Greg Myers), University of Lancaster: John Benjamins Publishing Company.

Achugar, Mariana, 2009: "Remembering and explaining a traumatic past: the Uruguayan military's narrative about the dictatorship" en John E. Richardson y Ruth Wodak (eds.): Critical Discourse Studies 6 (4), 283-295.

Achugar, Mariana, Amparo Fernandez y Nicolás MoRALES, 2011: "(Re)presentando el pasado reciente: la última dictadura uruguaya en los manuales de historia”, Discurso \& Sociedad 5 (2), 196-229.

Achugar, Mariana, Amparo Fernandez y Nicolás MoRALES, 2013: "Re/constructing the past: how young people remember the Uruguayan dictatorship", 
Discourse \& Society, volume 24, issue 3, 263-286.

Achugar, Mariana y Mary J. Schleppegrell, 2005: "Beyond connectors: The construction of cause in history textbooks", Linguistics and Education 16 (3), 298-318.

Fairclough, Norman y Ruth Wodak, 1997: "Critical discourse analysis" en Teun A. van Dıık (ed.): Discourse Studies. A Multidisciplinary Introduction, vol. 2: Discourse as Social Interaction, 258-284, London: Sage.

Feez, Susan, Rick Iedema y Peter White, 2008: Media Literacy, NSW Adult Migrant Education Service.

Garton Ash, Timothy, 2000: "El presente como historia”, Claves de Razón Práctica 102.

Giudice, Jacqueline, 2010: "Interpretación y reconstrucción del período 1976-1983 en manuales de ciencias sociales argentinos" en Oscar AlbahaCa, Mirna Kahwan y Sergio Serrón (coords.): Memorias del V Congreso Internacional de la Cátedra UNESCO, Instituto Venezolano de Investigaciones Lingüísticas y Literarias Andrés Bello - Cátedra UNESCO.

Giudice, Jacqueline y Estela Moyano, 2011: "Género y formación de ciudadanos: la re-construcción del periodo 1976-1983 en manuales argentinos para la escuela primaria" en Teresa Oteiza y Derrin Pinto (coords.): (Re)construcción: discurso, identidad y nación en los manuales escolares de historia y ciencias sociales, Santiago de Chile: Editorial Cuarto Propio, 205-268.

Halliday, Michael A. K., 1978: Language as a Social Semiotic, London: Edward Arnold.

Halliday, Michael A. K. y Christian Matthiessen, 2014: Introduction to functional grammar, cuarta edición, London y New York: Routledge.

KaPLAN, Nora, 2004: "Nuevos desarrollos en el estudio de la evaluación en el lenguaje: Ia teoría de la valoración”, Boletín de Lingüística 22, 52-78.
LEMKE, Jay L., 1998: "Resources for attitudinal meaning: evaluative orientations in text semantics", Functions of Language 5.1, 33-56.

Martin, James R., 2000: "Beyond exchange: Appraisal systems in English" en Evaluation in text: Authorial stance and the construction of discourse, Oxford: Oxford University Press, 142-175.

Martin, James R. y David Rose, 2008: Genre relations: Mapping culture, London: Equinox.

Martin, James R. y Peter R. R. White, 2005: The Ianguage of evaluation: Appraisal in English, London y New York: Palgrave Macmillan.

Oteiza, Teresa, 2003: "How Contemporary History is Presented in Chilean Middle School Textbooks", Discourse \& Society 14(5), 639-660.

Oteiza, Teresa, 2009a: "Solidaridad ideológica en el discurso de la historia: Tensión entre orientaciones monoglósicas y heteroglósicas", Revista Signos 42 (70), 219-244.

OteízA, Teresa, 2009b: "Evaluative patterns in the official discourse of Human Rights in Chile: giving value to the past and building historical memories in society". Edición especial de ponencias plenarias y mesas redondas del Cuarto Congreso de la Asociación de Lingüística Sistémico Funcional de América Latina, Universidade Federal de Santa Catarina, Florianópolis, Brasil. Delta 25, número especial, 609-640.

OteízA, Teresa, 2011: "Representación de las memorias del pasado: intersubjetividad en el discurso pedagógico de la historia" en Teresa Oteiza y Derrin Pinto (eds.): En (re)construcción: Discurso, identidad y nación en los manuales escolares de historia y de ciencias sociales, Santiago: Cuarto Propio, 129-172.

Oteiza, Teresa y Derrin Pinto, 2008: "Agency, Responsibility and Silence in the Construction of Contemporary History in Chile and Spain", Discourse \& Society 19 (3), 333-358.

Oteiza, Teresa y Derrin Pinto (eds.), 2011: En (re) construcción: discurso, identidad y nación en los manuales escolares de historia y ciencias socia- 
les, Santiago de Chile: Editorial Cuarto Propio.

Pascual, Mariana, 2010: "Potenciando la conexión entre el ACD y la LSF por medio de la Teoría de la Valoración: una exploración del discurso mediático argentino", comunicación presentada en el VI ALSFAL, Conferencia de la Asociación Latinoamericana de Lingüística Sistémico Funcional, Fortaleza, Brasil.

Pascual, Mariana, 2011: "La representación discursiva de las organizaciones de derechos humanos frente al discurso menemista: 'más allá de los costos y de los errores'”, comunicación presentada en el Congreso Internacional de Discursos y Medios, mesa redonda Medios, Discurso y Memoria Social, Buenos Aires, Argentina, del 19 al 21 de septiembre del 2011.

Pascual, Mariana, 2014: El empoderamiento en el aula de lenguas extranjeras: una propuesta desde el Análisis Crítico del Discurso y la Teoría de la Valoración, Lenguaje 42, número 1, 71-98.

ReIsIGL, Martin y Ruth Wodak, 2001: Discourse and discrimination, London: Routledge.

Shiro, Martha, 1998: Los pequeños cuentacuentos. El desarrollo de las habilidades narrativas de niños en edad escolar. Trabajo de ascenso inédito.

SHIRO, Martha, 2003: "Genre and evaluation in narrative development", Journal of Child Language 30, 165-195.

Vazquez, Inés, 2009 (ed.): Historia de las Madres de Plaza de Mayo, Ciudad Autónoma de Buenos Aires: Ediciones Madres de Plaza de Mayo.

White, Peter R. R., 1998: "Telling Media Tales: the News Story as Rhetoric”. Tesis doctoral, University of Sydney, Sydney.

White, Peter R. R., 2002: "Appraisal - The Language of Evaluation and Intersubjective Stance" [www. grammatics.com/appraisal, fecha de consulta: 24/04/2014].

Wodak, Ruth, 1996: Disorders of Discourse, London: Longman.
WodAk, Ruth, 2001: "The discourse-historical approach" en Ruth Wodak y Michael Meyer (eds.): Methods of critical discourse analysis, London: Sage, 63-95.

Wodak, Ruth y Michael Meyer, 2003: Methods of Critical Discourse Analysis, London: Sage.

Wodak, Ruth, Brigitte Mral y Majid KhosravinIK (eds.), 2013: Right Wing Populism in Europe: Politics and Discourse, London: Bloomsbury Academic.

Zullo, Julia, 2012: "Enseñar el Golpe: enunciadores, participantes y acontecimientos construidos para el aula" en Alejandro RaIter y Julia Zullo (coords.): Esclavos de las palabras, Buenos Aires: Editorial de la Facultad de Filosofía y Letras UBA. ZuLlo, Julia, 2014: "El golpe de Estado llega al aula. Las versiones para niños de la historia reciente", Discurso y Sociedad 8 (1), 12-36.

\subsection{Textos analizados}

"Incidentes en plaza de Mayo", diario El Tribuno, Salta, 10 de julio de 1985.

"Hubo serio incidente con Madres de Plaza de Mayo", diario Los Andes, Mendoza, 10 de julio de 1985.

"Dos lesionados como saldo de incidentes ocurridos antes y después del desfile”, diario La Capital, Rosario, 10 de julio de 1985.

"Hubo incidentes con las Madres de Plaza de Mayo", diario La Nación, Buenos Aires, 10 de julio de 1985.

"Incidentes entre las Madres y un grupo de civiles", diario Río Negro, General Roca, 10 de julio de 1985. 